УДК 622.24.051.64

\title{
СОВРЕМЕННЫЕ НАПРАВЛЕНИЯ СОВЕРШЕНСТВОВАНИЯ БУРОВОГО ИНСТРУМЕНТА ТИПА РDС
}

\author{
Нескоромных Вячеслав Васильевич1, \\ sovair@bk.ru
}

Лиу Баочанг²,

liubc@jlu.edu.cn

Чжаоран Чен ${ }^{2}$

564825155@qq.com

\section{Петенев Павел Геннадьевич 1 , pavel-whait@mail.ru}

\section{Попова Марина Сергеевна ${ }^{1}$, alleniram83@mail.ru}

\author{
Головченко Антон Евгеньевич1, \\ antong77@yandex.ru \\ 1 Сибирский федеральный университет, \\ Россия, 660095, г. Красноярск, пр. им. газеты «Красноярский рабочий», 95. \\ 2 Университет Цзилинь, \\ Китай, 130061, г. Чанчунь, ул. Химинжу, 938.
}

Актуальность. Требования к буровому породоразрушающему инструменту заключаются в обеспечении высокой механической скорости, ресурса, широкой области применения и небольших материальных затрат на его приобретение. Иными словами, буровой инструмент должен быть прочным, износостойким и изготовлен из относительно недорогого материала. Достижения в области синтеза поликристаллического алмаза и изготовления из него резцов высокой прочности значительно расширили возможности производителей бурового инструмента. Синтетический сверхтвердый материал стали применять при изготовлении режущих элементов и всей рабочей части породоразрушающего инструмента. Новшества материаловедения позволили производить резцы типа PDC разной формы, размеров, прочности, что особо актуально при создании долот, коронок и буровых головок с комбинированным вооружением. Как известно, состав и условия спекания композита влияют на свойства получаемой заготовки резца PDC. Изменяя прочностные параметры и геометрию расположения режущих элементов PDC с учетом области применения инструмента, можно добиться наилучшего эфффекта разрушения горной породы. Таким образом, при создании нового бурового породоразрушающего инструмента актуальным является объединение знаний и умений в области химии, материаловедения и конструирования, что приведет к получению перспективного породоразрушающего инструмента.

Цель: определить направления совершенствования бурового инструмента типа PDC, а именно возможности материаловедения в области спекания сверхтвердого материала $P D C$, преимущества применения композита различного состава при проектировании новых конструкций бурового инструмента типа PDC.

объекты: состав материала, процесс спекания и получаемые свойства резцов PDC, конструктивные особенности бурового инструмента PDC.

Методы: аналитические исследования, эксперимент, анализ.

Результаты. Изменение состава композита позволяет регулировать прочностные свойства получаемого резиа РDC. Добавление такого материала, как графен, повышает прочностные, тепло- и электропроводные свойства резца PDC. Объединение возможностей материаловедения и опыта конструирования позволяет добиться положительных результатов в области разработки нового перспективного породоразрушающего инструмента.

\section{Ключевые слова:}

Породоразрушающий инструмент, сверхтвердые материалы, поликристаллический алмаз, синтез, прочность, резец РDC, графрен.

\section{Введение}

Приоритетным направлением в развитии технических средств бурения является совершенствование породоразрушающего инструмента. Многие достижения в этой области связаны с использованием современных сверхтвердых материалов, созданием новых композиционных материалов, разработкой конструкций бурового инструмента с комбинированным вооружением [1-12].
В настоящее время наибольшие объемы бурения различного назначения связаны с использованием породоразрушающего инструмента типа $P D C$ (polycrystalline diamonds cutters). Ресурс такого инструмента в зависимости от геологических условий может достигать несколько сотен и более метров, а механическая скорость бурения в среднем составляет около 20-40 м/ч.

Резец $P D C$ получается путем спекания порошка поликристаллического алмаза на подложке в услови- 
ях очень высоких температур и давления (метод HTHP (high-temperature, high-pressure)). Для повышения прочностных характеристик и ускорения процесса спекания в порошок поликристаллического алмаза добавляется катализатор из металла VIII группы (железо, кобальт, никель). К примеру, расплавленный в условиях высокой температуры и высокого давления $(H T H P)$ кобальт катализирует частицы алмаза, поверхность которых графитизируется в процессе спекания. При растворении и осаждении алмаза между его частицами находится кобальт в жидком состоянии. Зерна алмаза соединяются друг с другом, в то время как кобальт мигрирует с образованием D-D связей между ними $[13,14]$. Однако под воздействием высокого давления частицы алмаза постепенно разрушаются, и большинство этих фрагментированных частиц остается в своих исходных состояниях. Таким образом, они не могут заполнить образовавшиеся промежутки между крупными зернами [15]. Кроме того, границы зерен разрушенных алмазных частиц не могут быть заполнены связкой $[16,17]$. Образовавшиеся пустоты между алмазами негативно сказываются на прочности, ресурсе резца и работе инструмента в целом.

Современное разнообразие материальной базы позволяет решить ряд проблем, связанных со снижением прочностных и других свойств композитных материалов. Известно, что для улучшения характеристик керамических материалов используется материал графен [18-20]. Он представляет собой гибкий, пластичный материал. Преимущественным свойством графена является возможность образования пленки на границе трения, что способствует хорошему эффекту самосмазки многокомпонентного материала. Присутствие графена вызывает рассредоточение напряжения сдвига в поверхностном слое, что приводит к пластической деформации на изнашиваемой границе раздела [21]. Поэтому графен часто добавляется в композит для достижения желаемого эффекта упрочнения [22-26].

Не стоит забывать и том, что графен является модификацией углерода, который также является химической основой синтетического поликристаллического алмаза. Именно поэтому данный материал выбран для рассмотрения в качестве составляющего элемента поликристаллического алмазного резца.

\section{Материалы и метод исследования}

С целью исследования влияния состава композита на свойства получаемого из него резца $P D C$ предложено провести сравнительный анализ образцов, спеченных из композита с добавлением графена и без него.

Для равномерного распределения фазы связки и полноценного заполнения пустот в материале при создании резцов $P D C$ с добавкой графена в составе композита предложено использовать поликристаллические синтетические алмазы различного размера. Гранулометрический состав алмазного порошка выглядел следующим образом: размер основных частиц - 30-50 мкм, размер сырых микронных частиц 4-8 мкм и размер мелких микронных частиц 1-2 мкм. При этом их весовое соотношение $\mathrm{W}_{30-50}: \mathrm{W}_{4-8}: \mathrm{W}_{1-2}$ соответственно составило 70:15:15. В качестве связующего элемента принят порошок кобальта $\left(\mathrm{C}_{0}\right)$. В качестве подложки использован карбид вольфрама $W C$-16. Путем ультразвукового диспергирования к графену, толщиной 6-8 нм и шириной 5 мкм, добавлен поливинилпирролидон.

Смешивание алмазного порошка (85-95 мас. \%), порошка кобальта (4-10 мас. \%) и графена $(0,05-0,3$ мас. \%) осуществлялось шаровой мельницей в течение 8 ч. После полного испарения спирта смешанный порошок обрабатывался в вакуумной печи при давлении $3,0 \times 10^{-3}$ Па и температуре $1000{ }^{\circ} \mathrm{C}$ в течение 6 ч для удаления примесей. После вакуумной обработки смесь помещалась в графитовую капсулу, а затем подвергалась высокотемпературной обработке под давлением в 5-6 ГПа и при температуре $1350-1600{ }^{\circ} \mathrm{C}$.

Полученные образцы материала подверглись сканирующей электронной микроскопии (SEMсканированию) (рис. $1, a, b)$. Как видно на рис. 1, во время спекания графен обволакивает поверхность алмазных частиц с образованием пленки на границе раздела фаз трения. В результате графен заполнил образовавшиеся промежутки между частицами алмаза и уменьшил сопротивление трения между ними, тем самым увеличивая компактность материала.

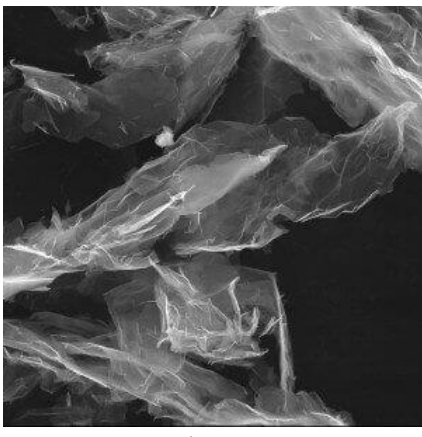

$a / a$

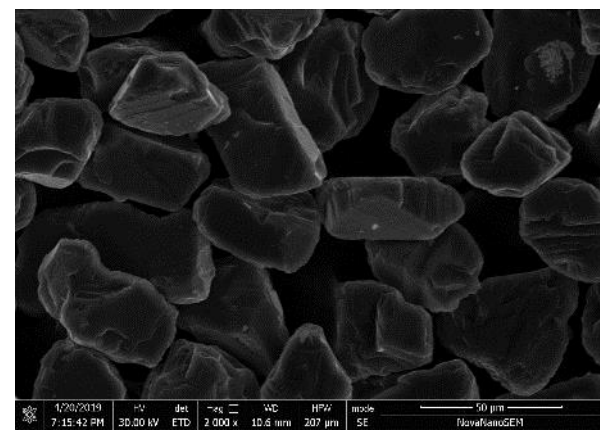

$\sigma / b$

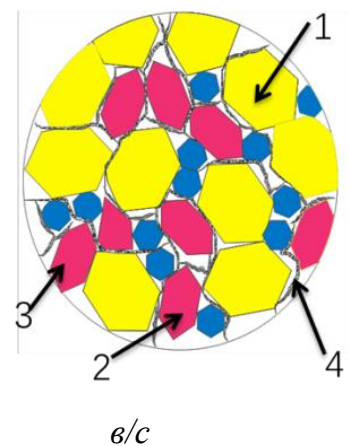

$B / c$

Pис. 1. Снимки SEM-сканирования композиционного материала: а) графен; б) алмаз; в) схема состава спеченного композита с добавкой графена: 1-алмазы размером 30-50 мкм, 2-4-8 мкм, 3-1-2 мкм, 4- графен

Fig. 1. SEM micrographs: a) graphene; b) diamond; c) size distribution of diamond particles and graphene: 1 - 30-50 $\mu$,

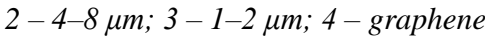


Полученная стабильность и компактность материала может послужить повышению прочности и износостойкости резца $P D C$.

На основе разработанного материала удалось изготовить резцы цилиндрической формы высотой 10 мм и диаметром 27 мм (рис. 2).

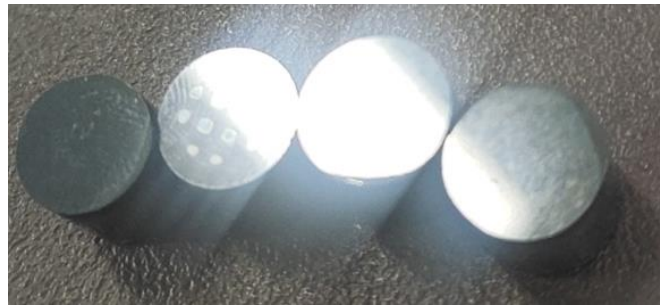

Pис. 2. Образеи РDC-графена

Fig. 2. PDC-graphene sample

\section{Анализ}

Для сравнительного анализа проведена серия лабораторных исследований свойств полученных образцов резца $P D C$. Условия температуры, давления и процесса спекания всех образцов приняты аналогично изготов- лению серийно-выпускаемого резца $P D C$. Одна группа образцов содержала графен и кобальт без дополнительных добавок (Graphene -Co), а другая группа coдержала только графит и кобальт (Graphite-Co).

Для выявления связующего состояния в образцах проведено SEM-сканирование, результаты которого (рис. 3) показали, что в образце Graphite-Co образовались кристаллы алмазов (рис. 3, a), а в образце Graphene-Co монокристаллы алмаза не были обнаружены (рис. 3, б).

С целью определения структурного состава образцы подверглись рентгеноструктурному $(X R D)$ и комбинационному анализу. Результаты $X R D$-исследования (рис. 4, a) показывают, что после высокотемпературного воздействия графен сохранил свои свойства в образце Graphene-Co.

Спектроскопия комбинационного рассеяния графита и графена до и после спекания НPHT (рис. 4, б) подтверждает, что графит в спеченном образце Graphene-Co преобразовался в кристаллы алмаза (розовый пик при $1332 \mathrm{~cm}^{-1}$ указывает на пик алмазообразования, синий пик означает, что графен сохранил свои свойства после спекания НТНР).

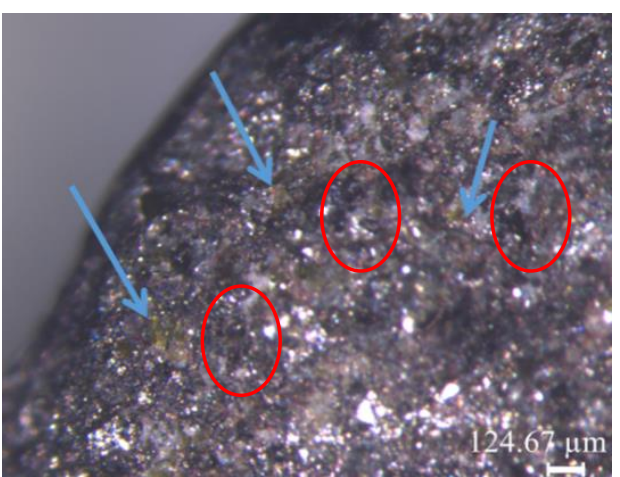

$a / a$

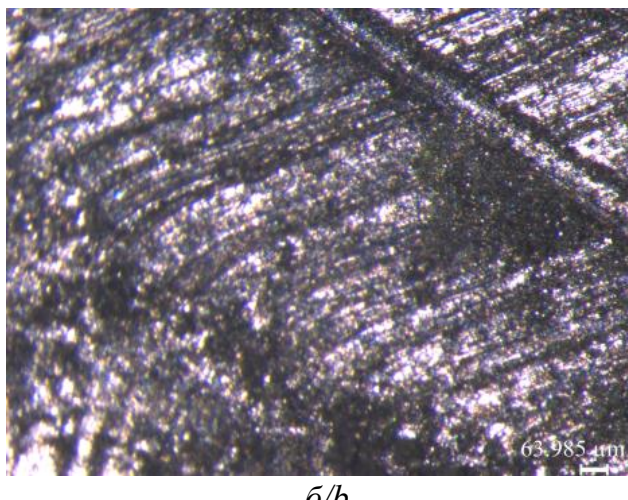

$\sigma / b$

Рис. 3. Результаты спекания материала при 6 ГПа и $1500{ }^{\circ}$ С: а) образеи Graphite-Со (кристалль алмаза указаны стрелками); б) образеи Graphene-Co

Fig. 3. a) diamond crystals (indicated by arrows) in the Graphite-Co sample at $6 \mathrm{GPa}$ and $1500^{\circ} \mathrm{C}$; b) no diamond single crystals in the Graphene-Co sample at $6 \mathrm{GPa}$ and $1500^{\circ} \mathrm{C}$
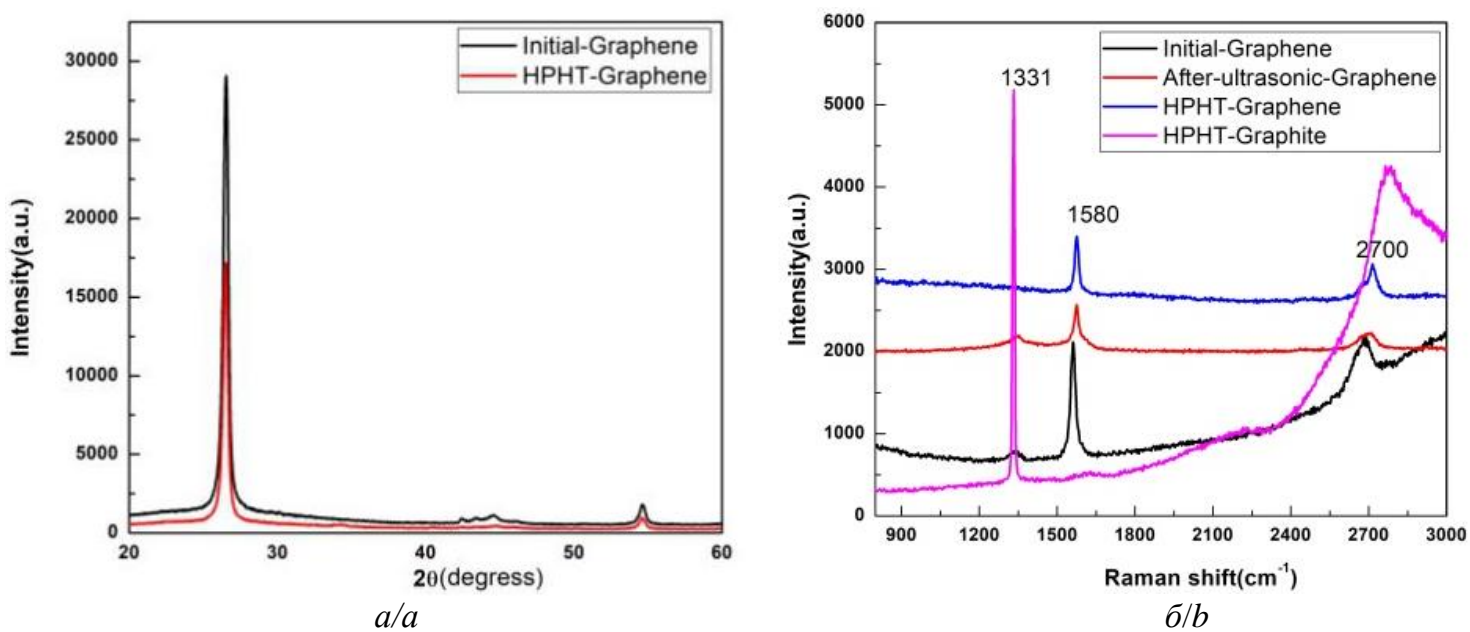

Puc. 4. Сравнительный анализ результатов исследования образиов Graphene-Co и Graphite-Co: a) XRD-cneктры; б) спектроскопия комбиначионного рассеяния графита и графена до и после спекания НРНТ

Fig. 4. XRD (a) and Raman (b) spectra of graphite and graphene before and after HPHT sintering 
Спеченный образец $P D C$, содержащий графен $(P D C-G)$, в основном состоит из алмаза, кобальта и карбида вольфрама. Проведенный $X R D$-анализ показал, что пиковое значение кристаллообразования алмазов в образце $P D C-G$ было более резким, чем в образце $P D C$ без графена. Этот результат указывает на лучшую кристаллизацию алмаза в $P D C-G$. Более того, в образце $P D C-G$ в пределах текущего разрешения графитовая фаза отсутствует, что указывает на то, что $P D C-G$ был изготовлен в стабильной зоне кристаллообразования алмазов. При этом в процессе спекания в образце $P D C$ $G$ графитизации не происходит, т. е. образец представляет собой чистый поликристаллический алмаз.

По уравнению (1) [27] можно определить связь между полученными спектрами комбинационного рассеяния и остаточными напряжениями в образце. Результаты расчетов показывают, что добавление некоторого количества графена значительно снижает интенсивность остаточных напряжений в $P D C-G$ (рис. 5). Интенсивность сжимающего напряжения, возникшего в середине поверхности $P D C$, постепенно уменьшается от центра к краям. Более того, на левой и правой кромках резца $P D C-G$ созданное напряжение сжатия изменилось на напряжение растяжения.

$$
\sigma=\left[v_{0}-v\left(\mathrm{~cm}^{-1}\right)\right] / 2,88,
$$

где $\sigma$ - остаточное напряжение (МПа); $v_{0}=1332 \mathrm{~cm}^{-1}$; $v$ - экспериментальный рамановский сдвиг в образце $P D C\left(\mathrm{~cm}^{-1}\right)$.
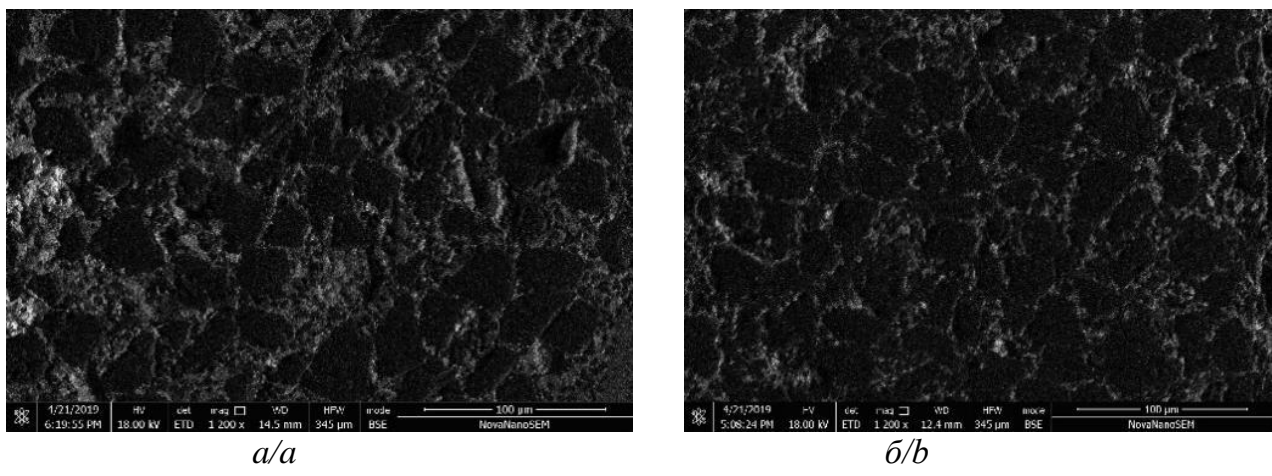

$\sigma / b$

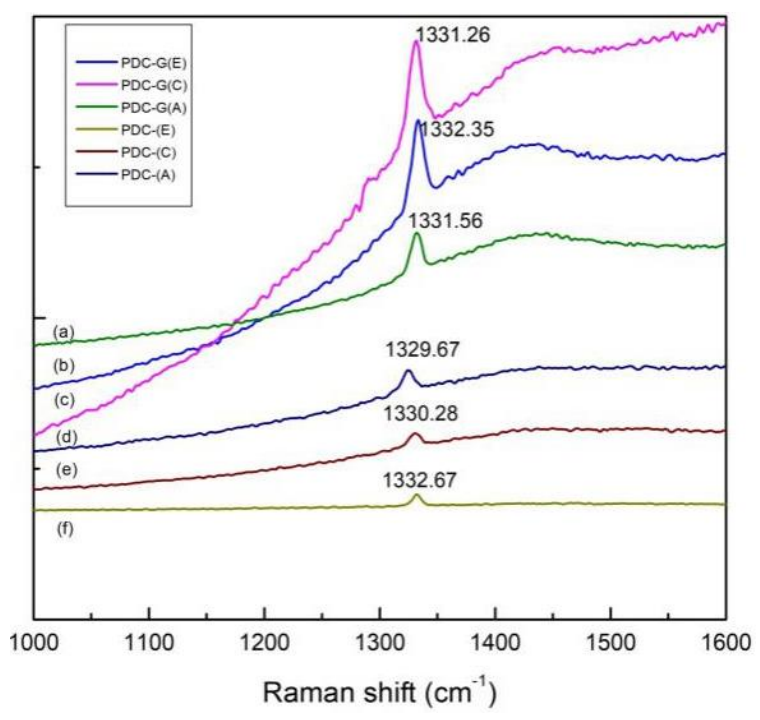

Puc. 5. Спектроскопия комбинационного рассеяния резиов РDC и PDC-G в крайних точках

Fig. 5. Raman shifts in $P D C$ and $P D C-G$ samples at extreme points

SEM-сканирование (рис. 6) показывает, что граница раздела поликристаллического алмаза, спеченного с графеном, была более плотной по сравнению с границей раздела $P D C$ без графена, при этом распределение частиц по размерам (15-20 мкм) было более равномерным [28].

Puc. 6. Кадры SEM-сканирования: а) резцы PDC; б) резиы PDC-G

Fig. 6. SEM images of PDC (a) and PDC-G samples (b)

Учитывая, что при спекании композита графен сохранил свои свойства, можно предположить, что он обеспечивает хорошую электро- и теплопроводность спеченного образца $P D C$.

С целью определения электропроводности графена в экспериментальных образцах использован четырехзондовый метод измерения [29]. Постоянный ток «I» пропускался через два внешних датчика, при этом между этими двумя датчиками потенциометром измерялось падение напряжения «V》. Coгласно уравнению (2) измеренные ток «I» и напряжение «V》 были преобразованы в значения сопротивления.

$$
\rho_{0}=\frac{V}{I} C
$$

$$
\text { где } C=\frac{20 \pi}{\frac{1}{S_{1}}+\frac{1}{S_{2}}-\frac{1}{S_{1}+S_{2}}-\frac{1}{S_{2}+S_{3}} \ldots \ldots . .} \text {. }
$$

Результаты исследования показали, что по сравнению с $P D C$ без графена электропроводность $P D C-G$ была улучшена примерно в 42 раза (рис. 7).

Для любого алмазного инструмента актуальным является вопрос теплопроводности.

Теплопроводность образцов $P D C$ и $P D C-G$ рассчитывалась по уравнению (3) $[10,29,30]$ :

$$
a=\frac{\lambda}{c \rho},
$$

где $a$ - коэффициент температуропроводности; $\lambda$ коэффициент теплопроводности; $c$ - удельная теплоемкость; $\rho$ - плотность. 


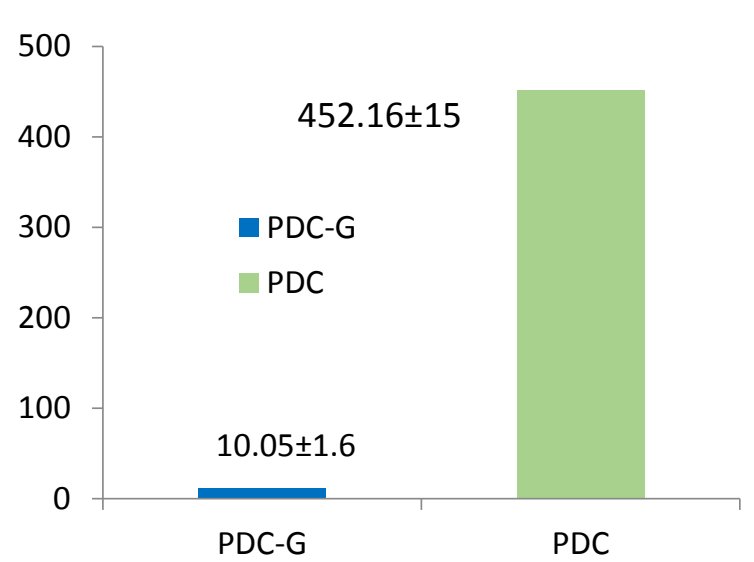

Puc. 7. Электропроводность резиов РDC и резиов $P D C-G$ Fig. 7. Electrical conductivity of PDC and PDC-G cutters

Результаты исследования теплопроводности показали, что с повышением температуры более выражено увеличение теплопроводности резцов $P D C-G$ (рис. 8) А при достижении температуры значений в $500 \mathrm{~K}$ коэффициент теплопроводности $P D C-G$ (с оптимальным содержанием графена) увеличился примерно на $60 \%$, что еще раз подчеркивает преимущество использования графена при изготовлении резцов $P D C$.

При всех вышеперечисленных достоинствах резцов $P D C-G$ не стоит забывать, что при проектировании бурового инструмента предпочтение во многом отдается механическим характеристикам резцов (прочность, износостойкость).

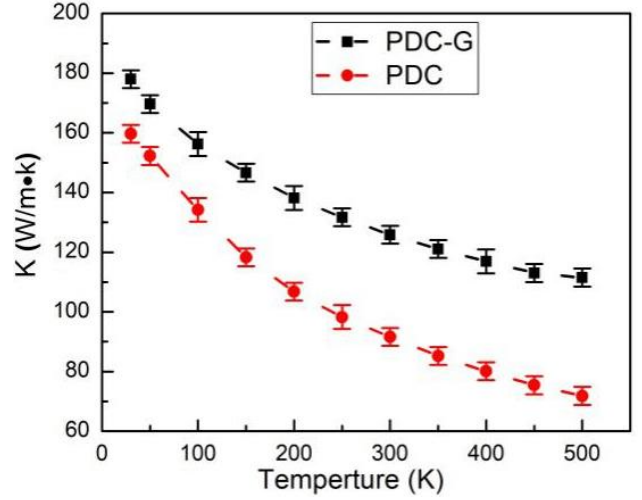

Puc. 8. Теплопроводность резиов $P D C$ и резиов $P D C-G$

Fig. 8. Thermal conductances of PDC and PDC-G cutters

Для более детального исследования механических свойств экспериментальных образцов $P D C-G$ применены два метода проверки износостойкости: с использованием шлифовального круга из карбида кремния и с использованием вертикального токарноревольверного станка (метод VTL) (сухой тест). Полученные в результате испытаний законы износостойкости по двум методам совпадают.

Согласно первому методу, при содержании графена 0,1 мас. \% он равномерно распределялся по поверхности алмазных частиц и по сравнению с $P D C$ без графена твердость и износостойкость $P D C-G$ с добавлением 0,1 мас. \% графена были улучшены на 75 и $33 \%$ соответственно (рис. 9).
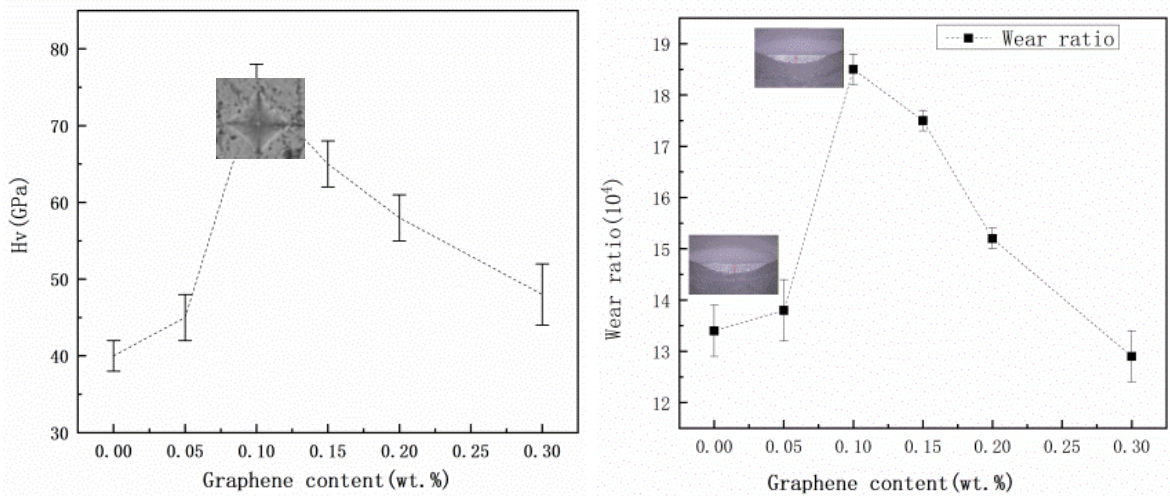

Pис. 9. Механические свойства резиов $P D C-G$ с различным содержанием графена

Fig. 9. Mechanical properties of PDC-G cutters with different graphene contents

По второму методу, когда шлифовальная часть $P D C$ невелика, степень износа составляет около 16 миллионов. Эксперимент показал, что по мере расширения шлифовальной горловины степень износа уменьшается. Коэффициент износа $P D C$, упрочненного графеном, согласно измерениям, - около 1,78 миллиона, а коэффициент износа $P D C$ - около 1,49 миллиона. Таким образом, по сравнению с обычным $P D C$ износостойкость $P D C$ с добавлением графена была увеличена на $18 \%$ (рис. 10).

\section{Выводы и заключения}

Таким образом, изменение состава композиционного материала может существенно повлиять на свойства и, как следствие, улучшить работу резца $P D C$, изготовленного из этого материала.

Так, внедрение одного лишь графена при неизменных условиях спекания резцов $P D C$ приводит к улучшению электропроводности примерно в 42 раза, повышению на 60 \% теплопроводности, улучшению твердости и износостойкости резца на 75 и $33 \%$ соответственно.

На сегодняшний день графен является не единственным представителем материалов, обладающих комплексом положительных качеств. К примеру, более перспективным, чем графен, ученые считают борофен, который по сравнению с графеном при меньшей толщине обладает большей прочностью. Подоб- 
ных материалов достаточно, и опыт применения их впечатляет. При изготовлении чувствительного к повышенным температурам алмазного инструмента особый интерес вызывает материал аэрогель, обла- дающий свойством высокой теплоизоляции. Полезной добавкой к композитным материалам может послужить прозрачный алюминий, прочность которого превышает прочность стали в три раза.
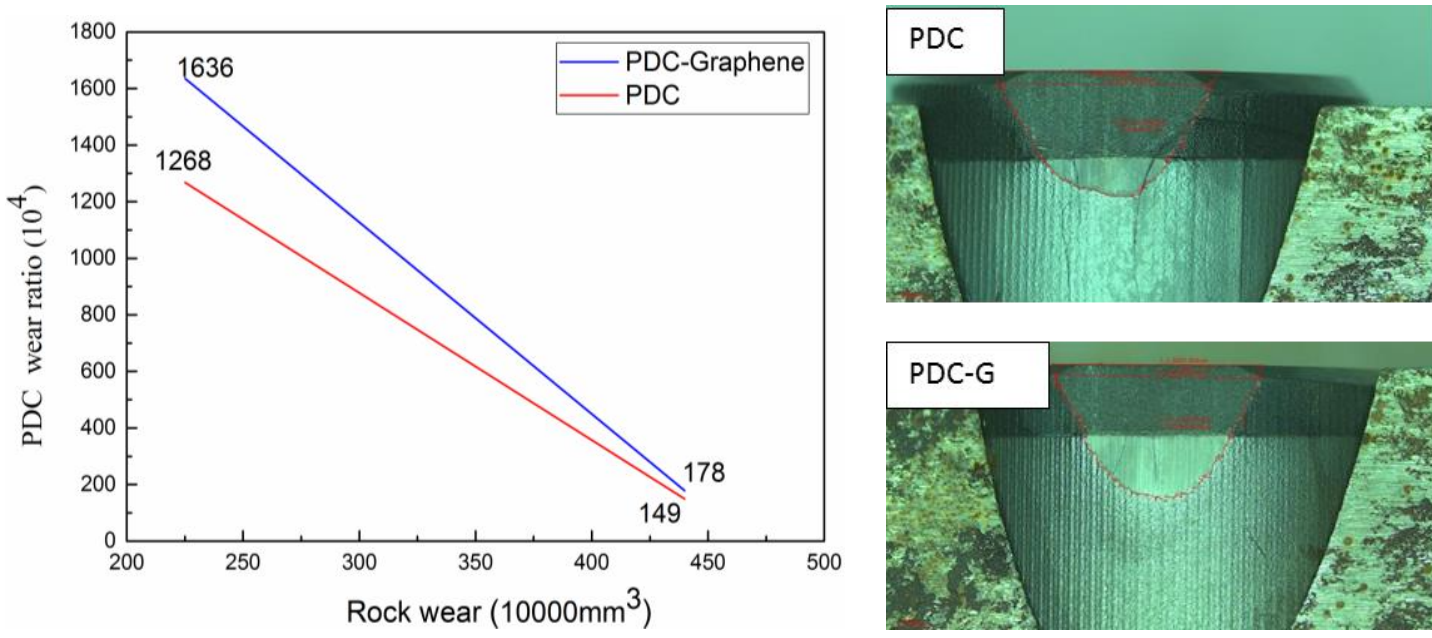

Puc. 10. Степень износа PDC и PDC-graphene по методу VTL

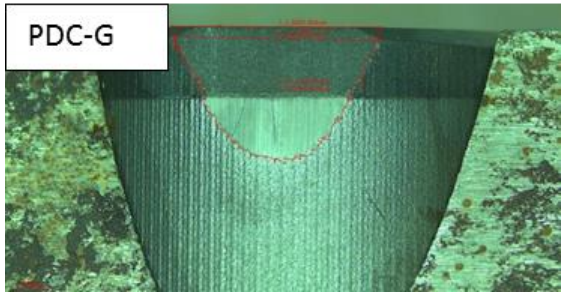

Fig. 10. VTL test wear ratio of $P D C$ and $P D C$-graphene

Необходимость комбинированного вооружения результат опыта разработки бурового инструмента, охватывающего расширенную область применения $[1,30]$. При изменении концентрации того или иного вещества (железо, кобальт, никель, графен, медь и т. д.) в составе композиционного материала появляется возможность регулировать свойства получаемых резцов $P D C$, не изменяя их геометрических параметров. Удобная цилиндрическая форма $P D C$ позволяет проектировать инструмент с максимальной динамизацией резцов, к примеру, так как это применено в долотах ONIX [4].

Учитывая такие преимущества резцов $P D C$, в Сибирском федеральном университете была разработана буровая коронка (патент РФ № 2715574) с вращаю- щимися резцами (рис. 11, a). Конструкция такой коронки не только способствует равномерному разрушению кольцевого забоя, но и увеличивает ресурс инструмента за счет равномерного износа режущей грани резцов $P D C$. Предложенная геометрия породоразрушающей части инструмента вполне позволяет размещать на дисках 1 и 2 комбинированное вооружение, что дает возможность расширить область применения бурового инструмента. В качестве резцов, размещенных на дисках, могут выступать материалы различной формы и качества, например, крупные синтетические алмазы, напыление поликристаллического алмазного порошка, резцы $P D C$ различного качества и т. д. Пример возможного вооружения показан на рис. 11,6 .
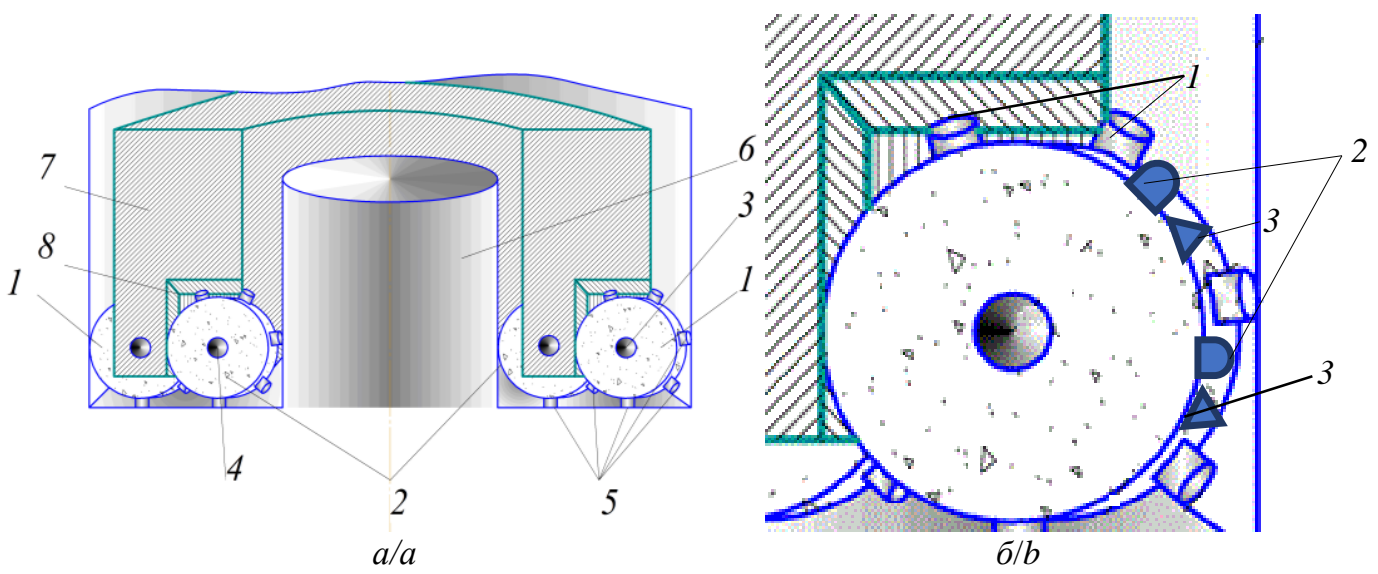

Pис. 11. Конструкиия коронки с вращающимися резиами PDC: а) общий вид в разрезе (1 - скважинообразующие диски; 2 - кернообразующие диски; 3, 4-оси вращения; 5-резиы PDC; 6-керн; 7 -матрица; 8 - окно) [31]; б) пример конструкции кернообразующих и скважинообразующих дисков с комбинированным вооружением (1-резцы PDC; 2 - крупный синтетический алмаз; 3 -резеи Tripax)

Fig. 11. Rotating incisor crown design PDC: a) general sectional view (1 - downhole discs; 2 - core discs; 3,4 - axis of rotation; 5 - cutter PDC; 6 - core; 7 - matrix; 8 -gap) [31]; b) example of the design of core-forming and wellforming discs with combined cutting (1 - cutter PDC; 2 -large synthetic diamond; 3 - cutter Tripax) 
Таким образом, применяя возможности материаловедения и опыт конструирования, можно добиться больших успехов в изготовлении бурового оборудования. К направлениям совершенствования инструмента типа $P D C$ следует отнести: повышение свойств износостойкости, прочности и температуропроводности и, как следствие, работоспособности.

При этом особое внимание следует уделять:

\section{СПИСОК ЛИТЕРАТУРЬ}

1. Бессон А., Берр Б., Диллард С. Новый взгляд на режущие элементы буровых долот // Нефтегазовое обозрение. - 2002. № 2. - C. 26-30.

2. Буренков Н.Н., Третьяк А.А., Чихоткин А.В. Режущая часть долота PDC: оптимизация геометрических параметров // Oil and Gas Journal Russia. - 2013. - № 5. - C. 56-58.

3. Инновационные подходы к конструированию высокоэффективного породоразрушающего инструмента / А.Я. Третьяк, В.В. Попов, А.Н. Гроссу, К.А. Борисов // Горный информационно-аналитический бюллетень. - 2017. - № 8. - С. 225-230.

4. Бакиев Р.Т., Пятницкий А.С. Применение PDC долот ONYX при бурении карбонатных и солевых отложений Ярактинского НГКМ в Иркутской области // Инженерная практика. - 2011. № 10. - С. 8-9.

5. Formation of pure polycrystalline diamond by direct conversion of graphite at high pressure and high temperature / T. Irifune, A. Kurio, S. Sakamoto, T. Inoue, H. Sumiya, K.-I. Funakoshi // Physics of the Earth and Planetary Interiors. - 2004. - № 143-144. P. 593-600.

6. Effect of removing internal residual metallic phases on wear resistance of polycrystalline diamond compacts, International / C. Liu, Z. Kou, D. He, Y. Chen, K. Wang, B. Hui, R. Zhang, Y. Wang // Journal of Refractory Metals and Hard Materials. 2012. - V. 31. - P. 187-191.

7. Belnap D., Griffo A. Homogeneous and structured PCD/WC-Co materials for drilling // Diamond and Related Materials. - 2004. V. 13. - № 10. - P. 1914-1922.

8. A novel method for measuring and analyzing the interaction between drill bit and rock / X. Wang, Z. Wang, D. Wang, L. Chai // Measurement. - 2018. - V. 121. - P. 344-354.

9. Guo D.Q., Hou C. Development of PDC drill bits for MWD directional drilling in underground coal mine // Procedia Earth and Planetary Science. - 2011. - V. 3. - P. 440-445.

10. Kanyanta V., Ozbayraktar S., Maweja K. Effect of manufacturing parameters on polycrystalline diamond compact cutting tool stressstate // International Journal of Refractory Metals and Hard Materials. - 2014. - V. 45. - P. 147-152.

11. Cutter layout optimization for reduction of lateral force on PDC bit using Kriging and particle swarm optimization methods / Y. Ma, Z. Huang, Q. Li, Y. Zhou, S. Peng // Journal of Petroleum Science and Engineering. - 2018. - V. 163. - P. 359-370.

12. A study on PDC drill bits quality / M. Yahiaoui, L. Gerbaud, J.Y. Paris, J. Denape, A. Dourfaye // Wear. - 2013. - № 298-299. P. 32-41.

13. Boland J.N., Li X.S. Microstructural characterisation and wear behaviour of diamond composite materials // Materials. - 2010. V. 3. - № 2. - P. 1390-1419.

14. Polycrystalline diamond compact with enhanced thermal stability / S. Liu, L. Han, Y. Zou, P. Zhu, B. Liu // Journal of Materials Science \& Technology. - 2017. - V. 33. - № 11. - P. 1386-1391.

15. Microstructure and properties of diamond/SiC composites prepared by tape-casting and chemical vapor infiltration process / Y. Liu, C. Hu, W. Feng, J. Men, L. Cheng, L. Zhang // Journal of the European Ceramic Society. - 2014. - V. 34. - № 15. P. 3489-3498.

16. Approach to controllable tribological properties of sintered polycrystalline diamond compact through annealing treatment / J. Li, W. Yue, W. Qin, C. Wang // Carbon. - 2017. - V. 116. P. 103-112.
1) улучшению качества процесса спекания материала резцов;

2) разработке и исследованию возможного состава композиционных материалов для изготовления резцов $P D C$;

3) разработке новых конструкций породоразрушающего инструмента типа $P D C$, позволяющих расширить область его применения.

17. Gu J., Huang K. Role of cobalt of polycrystalline diamond compact (PDC) in drilling process // Diamond and Related Materials. - 2016. - V. 66. - P. 98-101.

18. Graphene reinforced metal and ceramic matrix composites: a review / A. Nieto, A. Bisht, D. Lahiri, C. Zhang, A. Agarwal // International Materials Reviews. - 2016. - V. 62. - № 35. P. 241-302.

19. Mechanical properties and microstructure of a graphene oxidecement composite / Z. Pan, L. He, L. Qiu, A.H. Korayem, G. Li, J.W. Zhu, F. Collins, D. Li, W.H. Duan, M.C. Wang // Cement and Concrete Composites. - 2015. - № 58. - P. 140-147.

20. Yazyev O.V., Chen Y.P. Polycrystalline graphene and other twodimensional materials // Nature nanotechnology. - 2014. - V. 9. № 10. - P. 755-760.

21. The beneficial effect of graphene nanofillers on the tribological performance of ceramics / M. Belmonte, C. Ramírez, J. GonzálezJulián, J. Schneider, P. Miranzo, M.I. Osendi // Carbon. - 2013. № 61. - P. 431-435.

22. Shin J.-H., Hong S.-H. Fabrication and properties of reduced graphene oxide reinforced yttria-stabilized zirconia composite ceramics // Journal of the European Ceramic Society. - 2014. V. 34. - № 5. - P. 1297-1302.

23. Llorente J., Belmonte M. Friction and wear behaviour of silicon carbide/graphene composites under isooctane lubrication // Journal of the European Ceramic Society. - 2018. - V. 38. - № 10. P. 3441-3446.

24. Miranzo P., Belmonte M., Osendi M.I. From bulk to cellular structures: a review on ceramic/graphene filler composites // Journal of the European Ceramic Society. - 2017. - V. 37. - № 12. P. 3649-3672.

25. Tribological performance under dry sliding conditions of graphene/silicon carbide composites / J. Llorente, B. RománManso, P. Miranzo, M. Belmonte // Journal of the European Ceramic Society. - 2016. - V. 36. - № 3. - P. 429-435.

26. Фазовые превращения n-слойных графенов в алмаз при высоких давлениях и температурах / А.А. Шульженко, Л. Яворская, А.Н. Соколов, В. Гаргин, Н. Белявина // Журнал сверхтвердых материалов. - 2017. - V. 39. - № 2. - С. 75-82.

27. Residual stresses in sintered diamond-cobalt composites / H.A. Crostack, U. Selvadurai-Lassl, W. Tillmann, M. Gathen, C. Kronholz, T. Wroblewski, A. Rothkirch // Materials Science Forum. - 2006. - № 524-525. - P. 787-792.

28. Effect of diamond content on microstructure and properties of diamond/SiC composites prepared by tape-casting and $\mathrm{CVI}$ process / Y. Liu, C. Hu, J. Men, W. Feng, L. Cheng, L. Zhang // Journal of the European Ceramic Society. - 2015. - V. 35. № 8. - P. 2233-2242.

29. Yamashita M., Nishii T., Mizutani H. Resistivity measurement by dual-configuration four-probe method // Japanese Journal of Applied Physics 42. - 2003. - P. 1. - № 2A. - P. 695-699.

30. Case study. Kymera hybrid drill bit improves steerability and increases ROP $41 \%$ // Baker Hughes. 2020. URL: https://www.bakerhughes.com/case-study/kymera-hybrid-drill-bitimproves-steerability-and-increases-rop-41 (дата обращения 04.04.2021).

31. Буровая коронка: пат. 2715574 Рос. Федерация. заявл. 22.10.2019; опубл. 02.03.2020. - Бюл. № 7. - 6 c.

Поступила 19.04.2021 2. 


\section{Информация об авторах}

Нескоромных В.В., доктор технических наук, профессор, заведующий кафедрой технологии и техники разведки Института горного дела, геологии и геотехнологий Сибирского федерального университета.

Лиу Баочанг, профессор геоинженерии кафедры разведки и бурения, Университет Цзилинь, Китай.

Чжсаоран Чен, аспирант кафедры разведки и бурения, Университет Цзилинь.

Петенев П.Г., кандидат технических наук, доцент кафедры технологии и техники разведки Института горного дела, геологии и геотехнологий Сибирского федерального университета.

Попова M.C., кандидат технических наук, доцент кафедры технологии и техники разведки Института горного дела, геологии и геотехнологий Сибирского федерального университета.

Головченко A.E., ассистент кафедры технологии и техники разведки Института горного дела, геологии и геотехнологий Сибирского федерального университета. 
UDC 622.24.051.64

\title{
MODERN TRENDS IN DEVELOPMENT OF PDC-TYPE DRILLING TOOLS
}

\author{
Vyacheslav V. Neskoromnykh1, \\ sovair@bk.ru
}

Liu Baochang2,
liubc@jlu.edu.cn

Zhaoran Chen², 564825155@qq.com

Pavel G. Petenev1, pavel-whait@mail.ru

Marina S. Popova ${ }^{1}$, alleniram83@mail.ru

\author{
Anton E. Golovchenko', \\ antong77@yandex.ru \\ 1 Siberian Federal University, \\ 95, Krasnoyarskiy rabochiy avenue, Krasnoyarsk, 660095, Russia. \\ 2 Jilin University, \\ 938, Ximinzhu str., Changchun, 130026, China.
}

Relevance. Requirements for rock cutting tools are to ensure high mechanical speed, resource, wide range of application and low material costs for its purchase. In other words, the drilling tool must be durable, wear-resistant, and made of relatively inexpensive material. Advances in the synthesis of polycrystalline diamond and the manufacture of high-strength cutters from it have significantly expanded the capabilities of drilling tool manufacturers. Synthetic superhard material began to be used in the manufacture of cutting elements and the entire working part of rock cutting tools. Innovations in materials science have made it possible to produce PDC cutters of various shapes, sizes, strengths, which is especially important when creating bits, crowns and drill heads with combined weapons. As is known, the composition and sintering conditions of the composite affect the properties of the resulting PDC cutter blank. By changing the strength parameters and the geometry of the PDC cutting elements, taking into account the field of application of the tool, you can achieve the best effect of rock breaking. Thus, when creating a new drilling rock cutting tool, it is relevant to combine knowledge and skills in the field of chemistry, materials science and design, which will lead to obtaining a promising rock cutting tool.

The aim of the research is to determine the directions of improving the PDC-type drilling tool, namely, the possibilities of materials science in the field of sintering of superhard PDC material, the advantages of using a composite of various structure in the engineering of new designs of PDC-type drilling tools.

Objects: composition of material, sintering process and obtained properties of PDC cutters, design features of PDC drilling tools.

Methods: analytical research, experiment, analysis.

Results. Changing the structure of the composite allows you to adjust the strength properties of the resulting PDC cutter. Addition of such a material as graphene increases the strength, thermal and electrical conductive properties of PDC cutter. Combining the capabilities of materials science and design experience makes it possible to achieve positive results in the development of a new promising rock cutting tool.

\section{Key words:}

Rock cutting tool, superhard materials, polycrystalline diamond, synthesis, strength, PDC cutter, grapheme.

\section{REFERENCES}

1. Besson A., Burr B., Dillard S. Reinventing Drill Bit Cutters. Oil and Gas Review, 2002, no. 2, pp. 26-30.

2. Burenkov N.N., Tretyak A.A., Chikhotkin A.V. PDC bit cutter: geometric optimization. Oil and Gas Journal Russia, 2013, no. 5, pp. 56-58. In Rus.

3. Tretyak A.Ya., Popov V.V., Grossu A.N., Borisov K.A Innovative approaches to the design of highly efficient rock cutting tools. Mining Information and Analytical Bulletin, 2017, no. 8, pp. 225-230. In Rus.

4. Bakiev R.T., Pyatnitsky A.S. Primenenie PDC dolot ONYX pri burenii karbonatnykh i solevykh otlozheniy Yaraktinskogo NGKM $v$ Irkutskoy oblasti [Application of PDC bits ONYX when drilling carbonate and salt deposits of the Yarakta oil and gas condensate field in the Irkutsk region]. Inzhenernaya praktika, 2011, no. 10, pp. 8-9.

5. Irifune T., Kurio A., Sakamoto S., Inoue T., Sumiya H., Funakoshi K.-I. Formation of pure polycrystalline diamond by direct conversion of graphite at high pressure and high temperature. Physics of the Earth and Planetary Interiors, 2004, no. 143-144, pp. 593-600.

6. Liu C., Kou Z., He D., Chen Y., Wang K., Hui B., Zhang R., Wang Y. Effect of removing internal residual metallic phases on wear resistance of polycrystalline diamond compacts, International Journal of Refractory Metals and Hard Materials, 2012, no. 31, pp. 187-191.

7. Belnap D., Griffo A. Homogeneous and structured PCD/WC-Co materials for drilling, Diamond and Related Materials, 2004, no. 13 (10), pp. 1914-1922.

8. Wang X., Wang Z., Wang D., Chai L. A novel method for measuring and analyzing the interaction between drill bit and rock. Measurement, 2018, no. 121, pp. 344-354.

9. Guo D.Q., Hou C. Development of PDC Drill Bits for MWD Directional Drilling in Underground Coal Mine. Procedia Earth and Planetary Science, 2011, no. 3, pp. 440-445.

10. Kanyanta V., Ozbayraktar S., Maweja K. Effect of manufacturing parameters on polycrystalline diamond compact cutting tool stress- 
state, International Journal of Refractory Metals and Hard Materials, 2014, no. 45, pp. 147-152.

11. Ma Y., Huang Z., Li Q., Zhou Y., Peng S. Cutter layout optimization for reduction of lateral force on PDC bit using Kriging and particle swarm optimization methods. Journal of Petroleum Science and Engineering, 2018, vol. 163, pp. 359-370.

12. Yahiaoui M., Gerbaud L., Paris J.Y., Denape J., Dourfaye A. A study on PDC drill bits quality. Wear, 2013, no. 298-299, pp. 32-41.

13. Boland J.N., Li X.S. Microstructural characterisation and wear behaviour of diamond composite materials. Materials, 2010, no. 3 (2), pp. 1390-1419.

14. Liu S., Han L., Zou Y., Zhu P., Liu B. Polycrystalline diamond compact with enhanced thermal stability, Journal of Materials Science \& Technology, 2017, vol. 33, no. 11, pp. 1386-1391.

15. Liu Y., Hu C., Feng W., Men J., Cheng L., Zhang L. Microstructure and properties of diamond/SiC composites prepared by tape-casting and chemical vapor infiltration process Journal of the European Ceramic Society, 2014, vol. 34, no. 15, pp. $3489-3498$

16. Li J., Yue W., Qin W., Wang C. Approach to controllable tribological properties of sintered polycrystalline diamond compact through annealing treatment. Carbon, 2017, no. 116 , pp. 103-112.

17. Gu J., Huang K. Role of cobalt of polycrystalline diamond compact (PDC) in drilling process. Diamond and Related Materials, 2016, no. 66, pp. 98-101.

18. Nieto A., Bisht A., Lahiri D., Zhang C., Agarwal A. Graphene reinforced metal and ceramic matrix composites: a review. International Materials Reviews, 2016, 62, no. 5, pp. 241-302.

19. Pan Z., He L., Qiu L., Korayem A.H., Li G., Zhu J.W., Collins F., Li D., Duan W.H., Wang M.C. Mechanical properties and microstructure of a graphene oxide-cement composite, Cement and Concrete Composites, 2015, no. 58, pp. 140-147.

20. Yazyev O.V., Chen Y.P. Polycrystalline graphene and other twodimensional materials. Nature nanotechnology, 2014, vol. 9, no. 10 , pp. $755-760$

21. Belmonte M., Ramírez C., González-Julián J., Schneider J., Miranzo P., Osendi M.I. The beneficial effect of graphene nanofillers on the tribological performance of ceramics. Carbon, 2013, no. 61, pp. 431-435
22. Shin J.-H., Hong S.-H. Fabrication and properties of reduced graphene oxide reinforced yttria-stabilized zirconia composite ceramics. Journal of the European Ceramic Society, 2014, vol. 34, no. 5, pp. 1297-1302.

23. Llorente J., Belmonte M. Friction and wear behaviour of silicon carbide/graphene composites under isooctane lubrication. Journal of the European Ceramic Society, 2018, vol. 38, no. 10, pp. 3441-3446.

24. Miranzo P., Belmonte M., Osendi M.I. From bulk to cellular structures: a review on ceramic/graphene filler composites. Journal of the European Ceramic Society, 2017, vol. 12, no. 37, pp. 3649-3672.

25. Llorente J., Román-Manso B., Miranzo P., Belmonte M. Tribological performance under dry sliding conditions of graphene/silicon carbide composites. Journal of the European Ceramic Society, 2016, vol. 3, no. 36, pp. 429-435.

26. Shulzhenko A.A., Yaworskaya L., Sokolov A.N., Gargin V.G., Belyavina N.N. Phase transformations of n-layer graphenes into diamond at high pressures and temperatures, Journal of Superhard Materials, 2017, vol. 39, no. 2, pp. 75-82. In Rus.

27. Crostack H.A., Selvadurai-Lassl U., Tillmann W., Gathen M., Kronholz C., Wroblewski T., Rothkirch A. Residual stresses in sintered diamond-cobalt composites. Materials Science Forum, 2006, no. 524-525, pp. 787-792.

28. Liu Y., Hu C., Men J., Feng W., Cheng L., Zhang L. Effect of diamond content on microstructure and properties of diamond/SiC composites prepared by tape-casting and CVI process. Journal of the European Ceramic Society, 2015, no. 35 (8), pp. 2233-2242.

29. Yamashita M., Nishii T., Mizutani H. Resistivity measurement by dual-configuration four-probe method. Japanese Journal of Applied Physics 42, 2003, P. 1, no. 2A, pp. 695-699.

30. Case study. Kymera hybrid drill bit improves steerability and increases ROP $41 \%$. Baker Hughes. 2020. Available at: https://www.bakerhughes.com/case-study/kymera-hybrid-drill-bitimproves-steerability-and-increases-rop-41 (accessed 4 April 2021).

31. Neskoromnykh V.V., Vakhromeev A.G., Popova M.S., Bovin K.A., Kasintsev D.I., Parakhonko E.V Burovaya koronka [Drill bit]. Patent RF 2715574, 2020.

Received: 19 April 2021.

\section{Information about the authors}

Vyacheslav V. Neskoromnykh, Dr. Sc., professor, head of the department of technology and equipment of investigation, Siberian Federal University.

Liu Baochang, professor, Jilin University.

Zhaoran Chen, PhD student, Jilin University.

Pavel G. Petenev, Cand. Sc., associate professor, Siberian Federal University.

Marina S. Popova, Cand. Sc., associate professor, Siberian Federal University.

Anton E. Golovchenko, assistant, Siberian Federal University. 